\title{
Discrete Inverse Transformation for Eddy Current Tomography
}

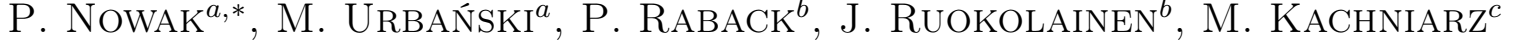 \\ AND R. SZEWCZYK ${ }^{a}$ \\ ${ }^{a}$ Institute of Metrology and Biomedical Engineering, Warsaw University of Technology, \\ Boboli 8, 02-525, Warsaw, Poland \\ ${ }^{b} \mathrm{CSC}$ - IT Center for Science, Keilaranta 14,FI-20101 Espoo, Finland \\ ${ }^{c}$ Industrial Research Institute for Automation and Measurements PIAP, \\ Al. Jerozolimskie 202, 02-486, Warsaw, Poland
}

\begin{abstract}
Paper presents results of a discrete inverse tomography transformation on exemplary results from eddy current tomography setup. Eddy current phenomena is highly non-linear and measurement results are ill-posed function of distribution of physical properties of the matter (mostly electrical conductivity and magnetic permeability). Thus the inverse transformation (reconstruction of objects shape) is based on an optimization algorithm in which objects model is described as a discrete array. With the usage of Finite Element Method (FEM) tomography measurement process is reconstructed and modelling results are compared with the measurement.
\end{abstract}

DOI: 10.12693/APhysPolA.133.701

PACS/topics: 02.30.Zz, 87.63.Pn, 02.70.Dh, 81.70.-q

\section{Introduction}

Eddy currents are commonly used for a non-destructive tests (NDT) of elements during production process, mainly for the selection of the elements. Reference objects (validated with other measurement method) are measured and their signature response is obtained. Then tested objects are measured and their response is compared with reference objects response. Thus a selection of defective objects can be made.

On the other hand, this method does not provide information about the defects' size and location, and cannot be used for correction of production process. This gap is supplemented by the usage of eddy current tomography $[1,2]$. This method has typical advantages of eddy current testing (ease of use, simple hardware, no need of safety requirements) as well as possibility of determination of the defects size and location, as in other tomography methods [3].

Inverse problem in eddy current tomography is complicated, due to the nature of the eddy current phenomena. Induced voltage is an implicit function of materials parameters (magnetic permeability and electrical conductivity) as well as geometrical properties of the tested element. For this reason, inverse transformation is based on optimization of the objects model and FEM-based forward transformation [4] for the model. Previously reported attempts [5-7] were based on a simplified 2D model. In presented solution full 3-D model, basing on Whitney edge elements [8], was utilized.

*corresponding author; e-mail: p.nowak@mchtr.pw.edu.pl
Paper presents an attempt of the development of inverse transformation for eddy current tomography, based on a discrete algorithm.

\section{Algorithm description}

Developed optimization algorithm is based on discrete changes in objects model parameters. Two possible states were assumed - in each model point there can either be material (steel with proper physical parameters) or air.

The cross-section of the modelled element is described as two 16x16 arrays, representing the distribution of magnetic permeability and electrical conductivity in the object. Initial state of the material array is presented in Fig. 1a).
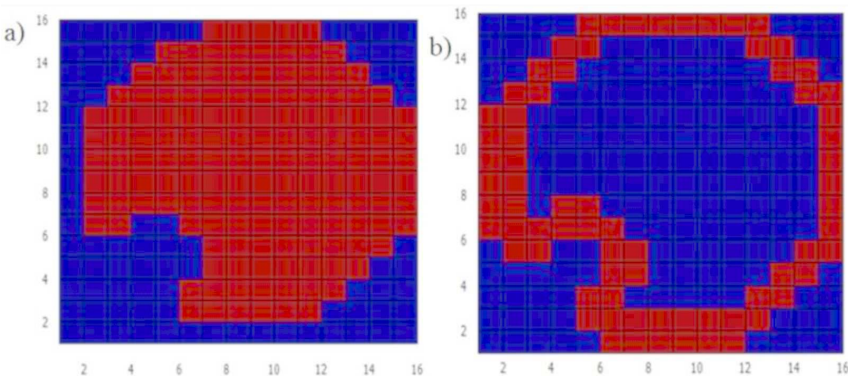

Fig. 1. a) Exemplary array of material distribution in the object b) Points on the material-air edge utilized for the optimization.

This model is used in FEM-based forward tomography transformation for selected measurement points $[9,10]$. The simulations are conducted for the high density mesh of the finite elements. FEM modelling requires data 
about the physical properties in all elements, so the values are interpolated from the modelled arrays. Presented algorithm does not require mesh generation for each modelling process. Meshes (for each linear position) are prepared before the optimization and consist of two coils representing the simplified tomography system, as well as cuboid, in which optimized array is inscribed.

After the FEM based forward transformation, modelling results are compared with the reference measurement results and quality factor is calculated. As a quality factor mean square error was selected. Afterwards algorithm randomly selects a point on the material-air edge, and changes its properties. An array of available points for the state presented in Fig.1 a) is shown in Fig.1 b). If a point was modelled as a material, its properties are set to the airs', and if it was modelled as air, its properties are set to the metals'.

After the FEM-based forward transformation for new model, new quality factor is calculated and compared with the previous one. If the quality factor is lower new model is taken for the next optimization step. If the quality factor is higher (changing the tested point did not improve the quality factor), algorithm changes back modified point and selects another one.

Optimization proceeds until no changes of points result with improvement of quality factor and optimization minimum is obtained. Lack of termination criterion for the presented algorithm ensures, that it will not stuck in local minimum.

\section{Obtained results}

Obtained modelling results for the algorithm result are presented in Fig. 2. Left-side graphs present result from FEM-based forward tomography transformation and right-side graphs present real measurement results, which were a reference to the optimization process. Top graphs present results of amplitude measure-

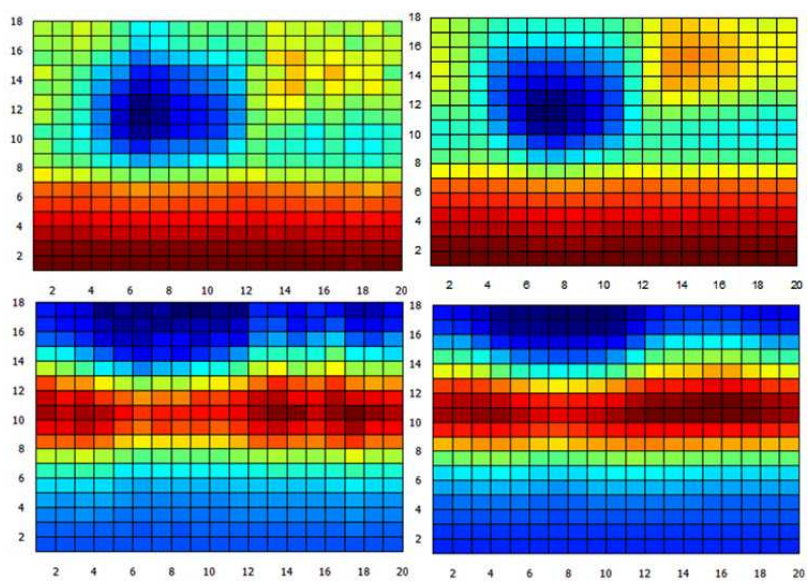

Fig. 2. Obtained modelling results (left graphs) compared with measurement results (right graphs). Results are presented in the function of linear position ( $Y$ axis) and objects rotation around its axis ( $X$ axis).

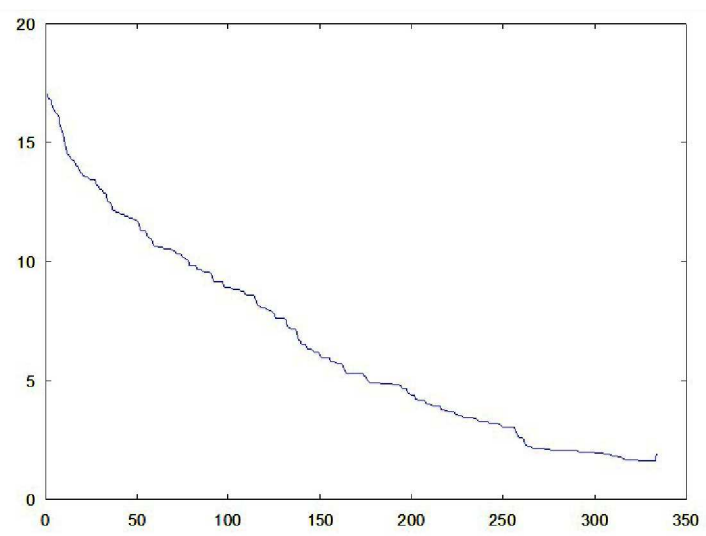

Fig. 3. Convergence graph for described algorithm.

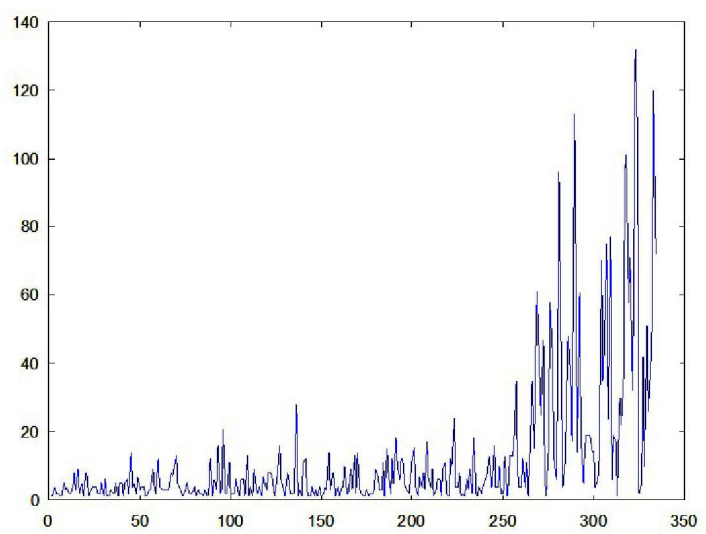

Fig. 4. Number of iterations in each optimization steps needed for quality factor improvement.

ment/modelling, whereas bottom graph present phase shift values.

High agreement of presented results is clearly visible, but modelling result present high level of numerical noise. This is caused by the transformation of Whitney edge elements to Cartesian space [4].

An analysis of the algorithm convergence was conducted. Results are presented in the Fig. 3. Continuous improvement of quality factor clearly confirms correctness of implemented algorithm. One may notice that after 250 optimization steps an improvement of quality factor is negligible.

In the Fig. 4 a number of iteration in each optimization step is presented. One may notice, that after 250 steps, the number of required optimization steps significantly increases. Taking that into account we may conclude, that proper selection of termination criterion will significantly improve the calculation time, without significant information loss.

Final results of material distribution in object are presented in Fig. 5. Colours represent the interpolated material distribution and black contour presents real object shape. One may notice, that algorithm removes the material inside the object, leaving mostly elements on the 


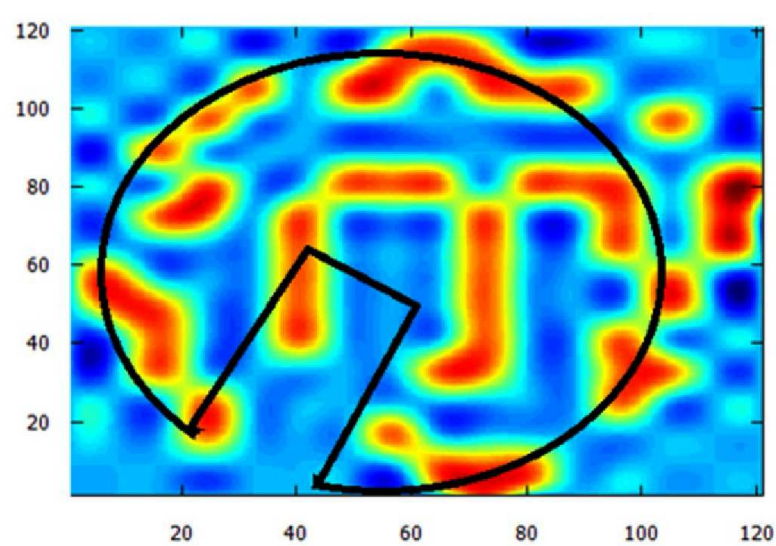

Fig. 5. Final results of algorithm - interpolated values of material distribution are presented in colour, whereas expected object shape is presented as a black contour.

edge. This is caused by the penetration depth of the eddy currents for the modelled frequency.

\section{Conclusions}

Paper presented an attempt of developing effective algorithm for inverse tomography transformation for eddy current tomography. Utilized algorithm was based on discrete optimization of modelled object, which was described as a material array. Obtained results are not satisfactory and presented method will not be further developed.

On the other hand, important knowledge may be obtained from the results analysis. Firstly, the termination criteria shall be applied for the optimization function. Data presented in Fig. 3 and 4 show, that algorithm proceeds with iterations, which are significantly timeconsuming but do not result with improvement of quality factor. Additionally, due to low depth of eddy current penetration, further algorithms shall consider mostly the optimization of modelled objects boundary.

Further work will concern development of continuous optimization algorithm with additional modification of objects magnetic permeability and electrical conductivity.

\section{Acknowledgments}

This work was partially supported by the statutory founds of Institute of Metrology and Biomedical Engineering, Warsaw University of Technology (Poland).

\section{References}

[1] J. Salach, in: 10.1007/978-3-319-10990-9 35 v. 317, Eds. J. Awrejcewicz, R. Szewczyk, M. Trojnacki, M. Kaliczyńska, Springer, Berlin 2015, p. 373.

[2] D. Premel, A. Mohammad-Djafari, IIEEE T. Magn. 31, 2000 (1995).

[2] F. Natterer, The Mathematics of Computerized Tomography, John Wiley \& Sons, New York 1986.

[3] R. Szewczyk, J. Salach, J. Ruokolainen, P. Råback, K. Stefko, M. Nowicki in: Advances in Intelligent Systems and Computing, v. 352, Eds. R. Szewczyk, C. Zieliński, M. Kaliczyńska Springer, Berlin 2015, p. 219.

[4] M. Soleimani, W.R.B. Lionheart, IEEE T. Med. Imaging, 25, 1521 (2006).

[5] M. Soleimani, W. Lionheart, A. Peyton, X. Ma in: Proceedings of the 3rd World Congress on Industrial Process Tomography, 2003, p. 252.

[6] M. Soleimani, C. N. Mitchell, R. Banasiak, R. Wajman, A. Adler, Prog. Electromagn. Res. 90, 171 (2009).

[7] A. Bossavit, IEE Proc. A, Phys. Sci., Meas. Instr., Manag. Edu. 135, 493 (1988).

[8] P. Nowak, R. Szewczyk, R. Ugodziński, P. Bazydło, in: Automation 2017 Innovations in Automation Robotics and Measurement Techniques, Eds. R. Szewczyk, C. Zieliński, M. Kaliczyńska, Springer, Berlin 2017, p. 481.

[9] P. Nowak, R. Szewczyk, in: PROCEEDINGS of the 21st International Conference on Applied Physics of Condensed Matter APCOM 2015, Eds. J. Vajda, I. Jamnicky, Bratislava 2015, p. 198. 